\title{
Solar vs. Fission Surface Power for Mars
}

\author{
Michelle A. Rucker \\ National Aeronautics and Space Administration, Lyndon B. Johnson Space Center, Houston, Texas, 77058
}

Steve Oleson, Pat George, Geoffrey A. Landis, James Fincannon, Amee Bogner, Robert E. Jones, and Elizabeth Turnbull

National Aeronautics and Space Administration, John H. Glenn Research Center, Cleveland, Ohio 44135

Michael C. Martini and John Z. Gyekenyesi, Zin Technologies, Cleveland, Ohio

Anthony J. Colozza, Paul C. Schmitz, and Thomas W. Packard Vantage Partners, LLC, Cleveland, Ohio

\begin{abstract}
A multi-discipline team of experts from the National Aeronautics and Space Administration (NASA) developed Mars surface power system point design solutions for two conceptual missions. The primary goal of this study was to compare the relative merits of solar- versus fission-powered versions of each surface mission. First, the team compared three different solar power options against a fission power system concept for a sub-scale, uncrewed demonstration mission. The 4.5 meter $(\mathrm{m})$ diameter pathfinder lander's primary mission would be to demonstrate Mars entry, descent, and landing techniques. Once on the Martian surface, the lander's In Situ Resource Utilization (ISRU) payload would demonstrate liquid oxygen propellant production using atmospheric resources. For the purpose of this exercise, location was assumed to be at the Martian equator. The three solar concepts considered included a system that only operated during daylight hours (at roughly half the daily propellant production rate of a round-the-clock fission design), a batteryaugmented system that operated through the night (matching the fission concept's propellant production rate), and a system that operated only during daylight, but at a higher rate (again, matching the fission concept's propellant production rate). Including $30 \%$ mass growth allowance, total payload masses for the three solar concepts ranged from 1,116 to $2,396 \mathrm{~kg}$, versus the $2,686 \mathrm{~kg}$ fission power scheme. However, solar power masses are expected to approach or exceed the fission payload mass at landing sites further from the equator, making landing site selection a key driver in the final power system decision. The team also noted that detailed reliability analysis should be performed on daytime-only solar power schemes to assess potential issues with frequent ISRU system on/off cycling. Next, the team developed a solar-powered point design solution for a conceptual four-crew, 500-day surface mission consisting of up to four landers per crewed expedition mission. Unlike the demonstration mission, a lengthy power outage due to the global dust storms that are known to occur on Mars would pose a safety hazard to a crewed mission. A similar fission versus solar power trade study performed by NASA in 2007 concluded that fission power was more reliable - with a much lower mass penalty - than solar power for this application. However, recent advances in solar cell and energy storage technologies and changes in operational assumptions prompted NASA to revisit the analysis. For the purpose of this exercise a particular landing site at Jezero Crater, located at $\mathbf{1 8}^{\circ}$ north latitude, was assumed. A fission power system consisting of four each $10 \mathrm{~kW}$ Kilopower fission reactors was compared to a distributed network of Orion-derived Ultraflex solar arrays and Lithium ion batteries mounted on every lander. The team found that a solar power system mass of about $9,800 \mathrm{~kg}$ would provide the 22 kilowatts $(\mathrm{kW})$ keep-alive power needed to survive a dust storm lasting up to 120-days at average optical depth of 5 , and $35 \mathrm{~kW}$ peak power for normal operations under clear skies. Although this is less than half the mass estimated during the 2007 work (which assumed latitudes up to $30^{\circ}$ ) it is still more than the $7,000 \mathrm{~kg}$ mass of the fission system which provides full power regardless of dust storm conditions. Relative merits-and
\end{abstract}


risks--of the two approaches were also evaluated. To assess latitude sensitivity, the team performed a brief assessment of a second notional landing site, selecting Columbus Crater located at $30^{\circ}$ south. Because dust storms do not appear to occur in the Southern hemisphere during the winter, when the days are shorter, solar array mass did not increase at Columbus Crater-but more battery mass would be required for the longer nights, and additional ISRU strings would be needed for optimal summer/winter cycling. Based on this work, it is clear that the choice between fission versus solar power for Mars surface systems is an important decision, with potential ramifications to crewed landing site selection. 